\title{
Le syndrome d'endométriose thoracique (TES). Une étiologie rare de pneumothorax itératifs chez la femme
}

\author{
Thoracic endometriosis syndrome: an uncommon etiology of iterative pneumothorax among \\ women
}

\author{
V. Jonquet $\cdot$ L. Chabot \\ Reçu le 21 février 2018; accepté le 4 avril 2018 \\ (C) SFMU et Lavoisier SAS 2018
}

\section{Introduction}

Les femmes sont moins exposées au pneumothorax spontané que les hommes (dans un rapport de 3 pour 1) mais sont plus sujettes aux pneumothorax secondaires, ont plus de récidives et plus de chirurgies [1]. Une des raisons avancées est l'endométriose thoracique [2]. Le cas exposé ci-dessous montre la découverte d'un syndrome d'endométriose thoracique révélé par un hydro-pneumothorax spontané.

\section{Observation}

Une femme de 32 ans sans antécédent d'origine africaine se présente aux Urgences pour douleur de l'hypochondre droit évoluant depuis plusieurs semaines. A l'examen clinique initial on note une hémodynamique stable et l'absence de fièvre. La palpation abdominale retrouve une douleur en hypochondre droit sans contracture. L'examen thoracique ne met pas en évidence de dyspnée et l'auscultation pulmonaire est dans les limites de la normale.

Sur le plan biologique : anémie modérée à $11,1 \mathrm{~g} / \mathrm{dL}$, VGM à $68 \mathrm{fl}$. Les leucocytes sont à 7,9 G/L et la CRP à $1,3 \mathrm{mg} / \mathrm{L}$. Le bilan hépatique est normal et le TP à $77 \%$. Le ionogramme sanguin est sans particularité et la clearance de la créatinine est à $133 \mathrm{~mL} / \mathrm{min}$.

Une échographie abdominale est alors demandée devant un tableau de colique hépatique hyperalgique. Cet examen élimine une pathologie des voies biliaires mais retrouve un

V. Jonquet $(\bowtie) \cdot$ L. Chabot

SAU-Samu-Smur CHG Laval (53),

Centre hospitalier de Laval,

33, rue du haut rocher, F-53000 Laval, France

e-mail : Vincent.jonquet@hotmail.fr épanchement intra-péritonéal ainsi qu'un épanchement pleural droit. Une radiographie du thorax confirme la présence d'un hydro-pneumothorax complet droit non compressif. Un scanner thoraco-abdomo-pelvien réalisé en urgence ne retrouve pas de lésions suspectes ni d'emphysème pulmonaire mais confirme la présence d'un hydropneumothorax droit et d'un épanchement intraabdominal (Fig. 1).

Un drainage thoracique est réalisé aux urgences et permet l'évacuation de l'épanchement gazeux ainsi que $1500 \mathrm{~mL}$ de liquide exsudatif séro-hémorragique dont l'examen cytobactériologique ne retrouve ni germes, ni de cellules malignes ni de bacilles acido-alcoolo-résistants.Une endométriose est suspectée devant les douleurs cycliques et la présence d'un hémothorax spontanée chez une femme en âge de procréer. L'exploration chirurgicale par thoracoscopie retrouve des adhérences et des brides stigmates d'épanchements antérieurs ainsi que de multiples taches rouille sur les deux plèvres et deux nodules diaphragmatiques. L'examen gynécologique retrouve un caractère cyclique des douleurs ainsi qu'un pelvis " figé » par les lésions d'endométriose et l'imagerie par résonnance magnétique confirme la présence des lésions d'endométrioses diffuses. L'analyse anatomopathologique des biopsies pleurales ne retrouve pas de cellules endométriales mais la présence de cellules musculaires lisses avec en périphérie des remaniements inflammatoires et hémorragiques ainsi que des follicules gigantocellulaires avec nécroses de coagulation et des infiltrats riches en macrophages et sidérophages. L'analyse immunohistochimique confirme l'absence de cellules endométriales mais les cellules musculaires lisses sont positives à la recherche de récepteurs à l'œstrogène et à la progestérone. Devant l'histoire clinique et la présence de lésion macroscopiquement évocatrice d'endométriose un traitement par leuproréline est débuté. La patiente est nettement améliorée par le traitement lors de la consultation de contrôle à 3 mois. 


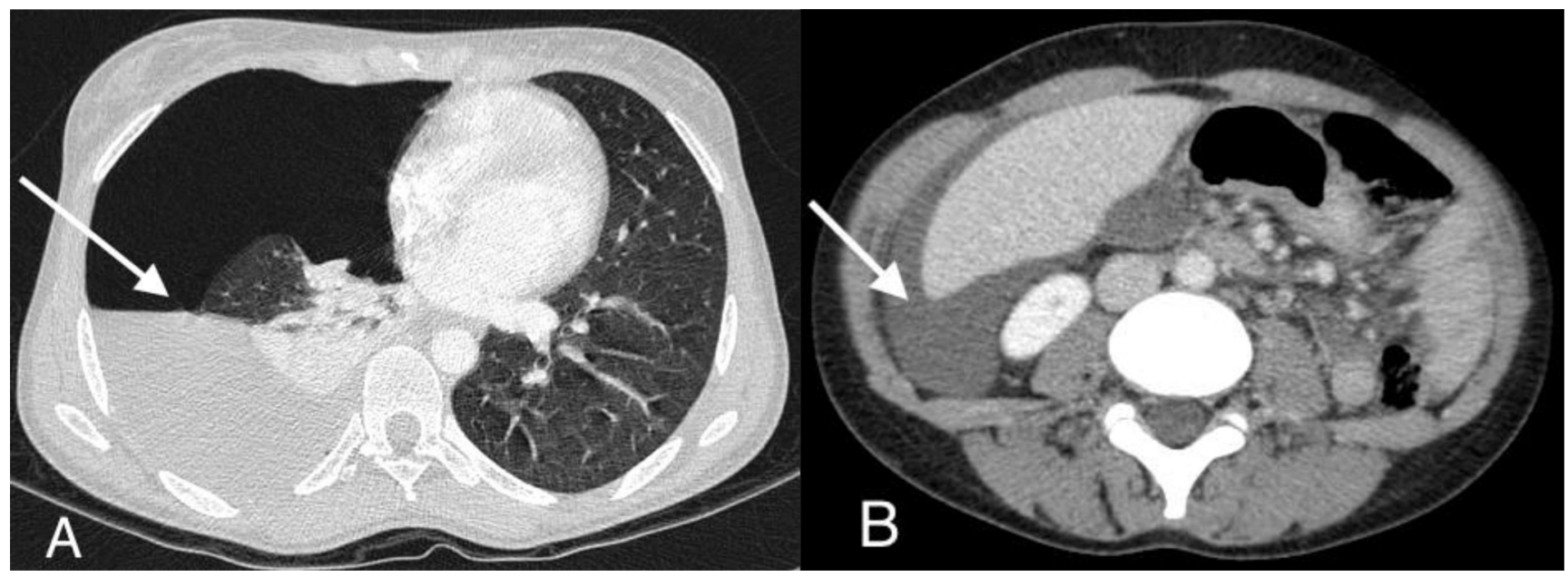

Fig. 1 A. Scanner thoraco-abdomino-pelvienne injecté temps portal montrant un hydropneumothorax droit de grande abondance (flèche). B. Coupe abdominale où l'on retrouve un épanchement intrapéritonéal péri hépatique de grande abondance (flèche)

\section{Discussion}

L'endométriose thoracique reste rare et peu décrite. Elle comprend quatre formes cliniques : le pneumothorax cataménial/l'hémothorax cataménial//'hémoptysie cataméniale/ nodule pulmonaire. Le pneumothorax cataménial qui représente la grande majorité des cas de syndrome d'endométriose thoracique correspond à 1 à $5 \%$ des cas de pneumothorax de la femme. Le caractère cataménial est défini par l'apparition du pneumothorax la veille et jusqu'à 72 heures après le début des menstruations. Cette pathologie se rencontre chez les femmes avec un âge moyen compris entre 30 et 34 ans. Le pneumothorax cataménial représente $73 \%$ des cas presque exclusivement à droite et $25 \%$ des pneumothorax spontanés de la femme nécessitant une prise en charge chirurgicale. L'hémothorax, les hémoptysies et les nodules constituent respectivement $14 \%, 7 \%$ et $6 \%$ des autres formes de syndrome d'endométriose thoracique. La présence d'un hémothorax et de nodule pulmonaires est souvent associée à une endométriose pelvienne [3]. Les pneumothorax sont partiels et les épanchements sont minimes en abondance.

Dans notre présentation la patiente présente une forme très rare d'endométriose du fait de l'abondance de l'épanchement thoracique et intra-péritonéal. Les présentations d'hydro-pneumothorax de grandes abondances associés à de l'ascite sont rarissimes et se rencontrent surtout chez la femme de race noire nullipare en âge de procréer. Elles doivent faire évoquer une pathologie néoplasique ou une tuberculose, mais des cas d'endométrioses avérés ont été décrits [4]. L'imagerie par résonnance magnétique est le meilleur examen pour la recherche d'endométriose pelvienne profonde avec une sensibilité et une spécificité de $90 \%$ et $91 \%$ [5]. Sur le plan anatomopathologique il est décrit dans la littérature la présence de tissu endométrial infiltrant ou non avec présence de cellules fusiformes et des stigmates d'hémorragies traduites par des sidérophages. L'immunohistochimie décrit des cellules positives à la vimentine et aux cellules musculaires lisses mais surtout une forte positivité aux récepteurs à l'œstrogène et la progestérone [6-7]. L'anatomopathologie n'est pas indispensable pour confirmer le diagnostic d'endométriose thoracique car peut faire défaut ou ne pas être contributive.

Le diagnostic a été posé sur l'histoire clinique des douleurs pelviennes et thoraciques rythmées par les règles et la présence de lésions pleurales évocatrices à la thoracoscopie. L'anatomopathologie conforta le diagnostic bien qu'on note l'absence de cellules endométriales. La patiente a bénéficié d'un traitement initialement chirurgical de l'hydropneumothorax puis traitée médicalement par leuproréline. Cette prise en charge semble validée mais les séries étudiées sont restreintes [8].

En conclusion l'endométriose thoracique est une étiologie rare d'épanchement thoracique. En revanche le clinicien, et ce même dès la prise en charge aux urgences, devrait évoquer ce diagnostic chez une femme dans sa troisième décennie qui présente des épanchements thoraciques droits récidivants afin de lui proposer une prise en charge adaptée.

Conflit d'intérêt : les auteurs déclarent ne pas avoir de lien d'intérêt.

\section{Références}

1. Nakamura H, Konishiike J, Sugamura A, et al (1986) Epidemiology of spontaneous pneumothorax in women. Chest 89:378-82 
2. Alifano M, Camilleri-Broët S (2008) Pneumothorax de la femme et endométriose thoracique. Rev Mal Respir 25:966-72

3. Joseph J, Sahn SA (1996) Thoracic endometriosis syndrome: new observation from an analysis of 110 cases. Am J Med 100:164-70

4. Flanagan KL, Barnes NC (1996) Pleural fluid accumulation due to intra-abdominal endometriosis: a case report and review of the literature. Thorax 51:1062-3

5. Bazot M, Darai E, Hourani et al (2004) Deep pelvic endometriosis: MR Imaging for diagnosis and prediction of extension of disease. Radiology 232:379-89
6. Flieder DB, Moran CA, Travis WD et al (1998) Pleuro-pulmonary endometriosis and pulmonary ectopic deciduosis : a clinicopathologic and immunohistochemical study of 10 cases with emphasis on diagnostic pitfalls. Hum Pathol 29:1495-503

7. Huber M, Wierrani F, Böhm G, et al (1999) Multiple endometrial stromal nodules with sparse cysts and glands in the lung a nodular variation of endometriosis that may mimic metastases of sarcoma. Pathol Res Pract 195:59-64

8. Alifano M, Trisolini R, Cancellieri A, et al (2006) Thoracic endometriosis: current knowledge. Ann Thorac Surg 81:761-9 